\title{
Premature newborn with intracardiac fungus balls and endocarditis which is curable only with anti-fungal therapy: Case presentation
}

\author{
Mecnun Cetin*, Serkan Ozen, Munevver Yildirimer, Murat Deveci, Senol Coskun, \\ Metehan Kizilkaya \\ Division of Pediatric Cardiology, Department of Pediatrics, Faculty of Medicine, Celal Bayar University, Manisa, Turkey; \\ *Corresponding Author: drmecnun@hotmail.com
}

Received 22 November 2012; revised 26 December 2012; accepted 3 January 2013

Copyright (c) 2013 Mecnun Cetin et al. This is an open access article distributed under the Creative Commons Attribution License, which permits unrestricted use, distribution, and reproduction in any medium, provided the original work is properly cited.

\begin{abstract}
Invasive fungal infections often caused by Candida species are infections which have high morbidity and mortality rates in newborn infants. $5 \%$ of the cases have also concomitant endocarditis and this causes mortality rates over $60 \%$. Thus it has vital importance to diagnose and treat these patients with endocarditis in early stages. Most preferred treatment option is a surgical incision in addition to systemic anti-fungal therapy. Here we presented an extremely low birth weight, premature newborn with endocarditis caused by Candida tropicalis and cured solely by systemic anti-fungal treatment without the need for surgical intervention.
\end{abstract}

Keywords: Invasive Fungal Infection; Endocarditis; Candida tropicalis

\section{INTRODUCTION}

Frequency of invasive fungal infection in newborns is increasing due to the reasons such as increasing number of low/extremely low birth weight newborns that are hospitalized in newborn intensive care units, increased invasive interventions, need for parenteral nutrition, use of broad-spectrum antibiotics and need for long term mechanical ventilation [1]. The most common infectious agent is $C$. albicans. These fungal infections develop severe symptoms in extremely low birth weight newborns and have mortality rates of $30 \%-60 \%$ [2]. Endocarditis develops in $5 \%$ of invasive Candida infections and these cases have over $60 \%$ mortality rate. In this article a case of extremely low birth weight newborn with endocarditis caused by Candida tropicalis and cured by only systemic anti-fungal therapy due to high risk of mortality with surgical intervention, anticoagulant and thrombolytic treatment is presented.

\section{CASE}

Female baby, who was born 980 gr. from 24-year-old mother's first pregnancy, with C-section at 28 weeks because of umbilical cord prolapse, had APGAR scores of 3 and 7 respectively at 1st and 5th minutes. After five hours, with onset of respiratory distress, grunting, apnea and presence of acidosis and hypoxia in her arterial blood gas, patient was intubated and connected to mechanical ventilator. Umbilical catheter was inserted and TPN solution and sefotaxim (100 mg/kg/day, two doses) plus amikacin (18 mg/kg/dose, in every 35 hours) was initiated. With the detection of reticulonodular pattern in PA chest radiography patient was diagnosed as respiratory distress syndrome and administered surfactant. Complete blood count and biochemical values of the patient were normal. On third day, stage III hemorrhage in left hemisphere was detected on transfontanel ultrasonography. On the fourth day, Patent Ductus Arteriosus (PDA) was found by transthoracic echocardiogram (TTE) that was performed upon detection of 2/6 grade systolic murmurs during cardiovascular system examination. Patient received three doses of intravenous ibuprofen (first dose of $10 \mathrm{mg} / \mathrm{kg}$ and subsequent two doses of $5 \mathrm{mg} / \mathrm{kg}$ ) with 24 hour intervals. In control echocardiogram performed one week later it was observed that PDA was closed. However at the interconnection of vena cava inferior and left atrium a hyperechoic mass (fungus ball) of $11.3 \mathrm{~mm} \times 5 \mathrm{~mm}$ (Figure 1) was spotted. Same day as yeast cell proliferation in previously collected blood culture sample was detected, abdominal catheter was removed and systemic liposomal amphotericin B therapy 


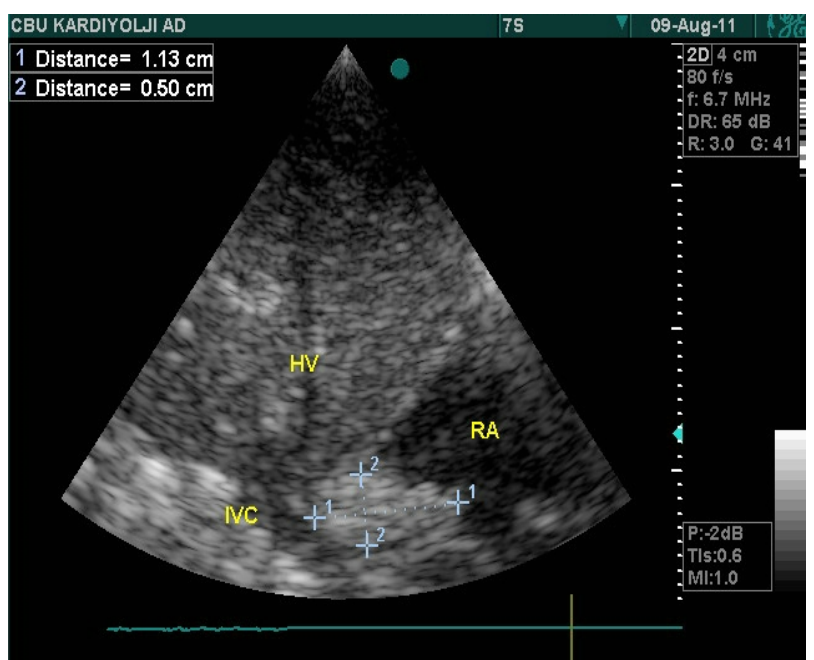

Figure 1. Appearance of the fungus ball at interconnection of vena cava inferior and right atrium (IVC: Inferior vena cava; HV: Hepatic vein; RA: Right atrium).

(6 mg/kg/day, one daily dose) was initiated. Later it was realized that proliferated agent in the blood culture was amphotericin B sensitive Candida tropicalis and same $C$. tropicalis proliferation was observed in both second blood culture and abdominal catheter culture. There was no proliferation in urine culture. Lumber puncture and eye examination was normal. Patient was intubated and extubated sporadically 3 times with recurrent severe apnea exacerbations during the follow-ups. Surgical excision was planned for patient's fungus ball in the vena cava inferior but patient's extremely low birth weight, unstable clinical condition and high cardio-pulmoner bypass risk postponed the surgery and only liposomal amphotericin B therapy was continued. We couldn't use anticoagulant and thrombolytic treatment due to presence of intracranial hemorrhage. In periodically obtained echocardiograms of patient during follow-ups, shrinkage of fungal mass was observed. At the 50th day of the antifungal treatment complete disappearance of the mass was observed (Figure 2) and treatment continued in following next 10 days. Patient was administered 60 days of anti-fungal therapy at total. There were no significant side effects related to anti-fungal therapy. After clinical stabilization was achieved during follow-ups, patient was discharged from hospital as 2100 gr.

\section{DISCUSSION}

Candida species can cause organ specific invasive infections aside from non-lethal local mucocutaneous infections. Most common infectious agents in invasive fungal infections are Candida species [3]. Recently increased number of nosocomial Candida infections among populations are reported all around the world. In 196070 's most of nosocomial candidasis cases were caused by

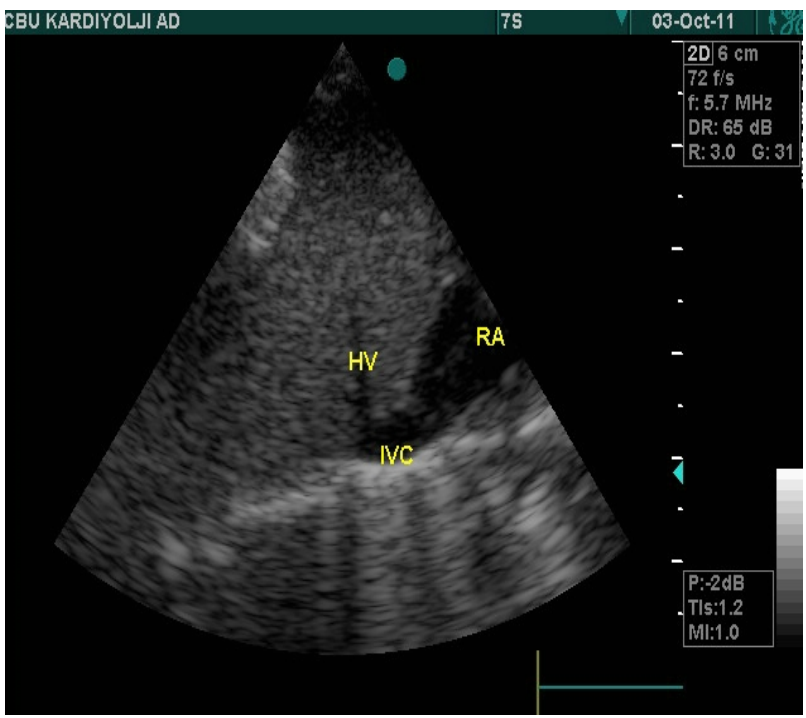

Figure 2. Disappearance of fungus ball (IVC: Inferior vena cava; HV: Hepatic vein; RA: Right atrium).

C. albicans however recently a significant increase in incidence of infections caused by non-albicans Candida species (C. tropicalis, C. glabrata, C. parapsilosis, $C$ krusei, C. guiliermondii, C. lipolytica, and C. pelliculosa) has been observed [4]. In our case $C$. tropicalis proliferation was observed in blood culture.

Risk factors for invasive candidiasis in neonatal period includes low gestational week, early membrane rupture, use of $\mathrm{H}-2$ receptor blockers in intensive care units, intubation and mechanical ventilation, the presence of central venous catheter, use of broad-spectrum antibiotics, lack of enteral nutrition and utilization of total parenteral nutrition $[5,6]$. Five of these risk factors were present in our patient.

Systemic fungal infection is reported in very low birth weight infants less than $1500 \mathrm{~g}$, as the third most frequent cause of late-onset sepsis and is associated with high mortality [7]. Fungal endocarditis is an uncommon complication of invasive candidial infection, but there is a reported increase in the incidence of such infection over the last 15 years $[8,9]$. Therefore, early recognition of infection and therapy is imperative.

Invasive candidiasis must definitely be considered in cases with extremely low birth weight premature newborns who have risk factors for candidiasis. Successful treatment of patients with endocarditis requires early recognition and aggressive treatment. The first and most basic principle of treatment is elimination of the source that is associated with increased risk for candidiasis and the second step is initiating appropriate anti-fungal therapy.

Management of fungal endocarditis in premature infants is controversial. Use of prolonged anti-fungal therapy in conjunction with surgical removal of thrombus is 
shown to be an effective approach to the treatment of this complication [10]. Use of prolonged anti-fungal treatment alone is reported in few critically ill infants with fungal endocarditis [11]. In our case, due to high risk of mortality for cardiopulmoner bypass, only 60 days liposomal amphotericin B therapy was administered. Due to intracranial hemorrhage risk no anticoagulant and thrombolytic therapy was used. We observed that intracardiac vegetation shrunk on the 20th day of the treatment and completely disappeared on the 50th day. There are few cases, similar to our case, in literature which are treated and cured by only anti-fungal therapy without the use of anticoagulants and thrombolytics and surgical intervention.

Another aspect that we would like to highlight is the prophylactic anti-fungal therapy in high risk extremely low birth weight group patients. Although this is a controversial topic, application of fluconazole prophylaxis for $<1000$ gr. newborns have grade A1 level of evidence in Infectious Diseases Society of America's (IDSA) Practice Guidelines and $3 \mathrm{mg} / \mathrm{kg}$ fluconazole should be applied parenterally and in two divided doses for at least 14 days starting from the first day of life [12]. Reason for us not initiating prophylaxis was due to low frequency of candidiasis cases in our center.

We suggest that prophylactic anti-fungal therapy is essential in cases with high risk factors for invasive candidiasis during neonatal period. Besides, serial echocardiographic evaluation of newborns with invasive candidiasis for possible cardiac involvement should definitely be performed.

As a result, solitary long-term systemic anti-fungal treatment can be a favorable treatment option in newborn patients with fungal endocarditis, such as the one in our case, where surgical intervention, anticoagulant or thrombolytic therapy due to their high risk.

\section{REFERENCES}

[1] Kaufman, D., Boyle, R., Hazen, K.C., Patrie, J.T., Robinson, M. and Donowitz, L.G. (2001) Fluconazole prophylaxis against fungal colonization and infection in preterm infants. New England Journal of Medicine, 345, 16601666. doi:10.1056/NEJMoa010494
[2] Zaoutis, T.E., Heydon, K., Localio, R., Walsh, T.J. and Feudtner, C. (2007) Outcomes attributable to neonatal candidiasis. Clinical Infectious Diseases, 44, 1187-1193. doi:10.1086/513196

[3] Lamagni, T.L., Evans, B.G., Shigematsu, M., et al. (2001) Emerging trends in the epidemiology of invasive mycoses in England and Wales (1990-9). Epidemiology \& Infection, 126, 397-414. doi:10.1017/S0950268801005507

[4] Trick, W. (2002) Secular trend of hospital-acquired candidemia among intensive care unit patients in the United States during 1989-1999. Clinical Infectious Diseases, 35, 627. doi:10.1086/342300

[5] Manzoni, P. (2006) Risk factors for progression to invasive fungal infection in preterm neonates with fungal colonization. Pediatrics, 118, 2359-2364. doi:10.1542/peds.2006-1311

[6] Benjamin, D.K. (2006) Neonatal candidiasis among extremely low birth weight infants: Risk factors, mortality rates, and neurodevelopmental outcomes at 18 to 22 months. Pediatrics, 117, 84-92. doi:10.1542/peds.2004-2292

[7] Stoll, B.J., Hansen, N., Fanaroff, A.A., Wright, L.L., Carlo, W.A., Ehrenkranz, R.A., et al. (2002) Late onset sepsis in very low birth weight neonates: The experience of NICHD Neonatal Research Network. Pediatrics, 110, 285-291. doi:10.1542/peds.110.2.285

[8] Kossoff, E.H., Buescher, E.S. and Karlowicz, M.G. (1998) Candidemia in Neonatal Intensive Care Unit: Trend during fifteen years and clinical features of 111 cases. Pediatric Infectious Disease Journal, 17, 504-508. doi:10.1097/00006454-199806000-00014

[9] Faix, R.G. (1984) Systemic candida infections in infants in intensive care nurseries, high incidence of central nervous system involvement. Journal of Pediatrics, 105, 616-622. doi:10.1016/S0022-3476(84)80433-3

[10] Ankola, P.A., Perveen, S. and Fish, B. (2006) Fungal endocarditis. Journal of Perinatology, 26, 509-510. doi:10.1038/sj.jp.7211557

[11] Zenker, P.N., Rosenberg, E.M., Van Dyke, R.B., Rabalais, G.P. and Daum, R.S. (1991) Successful medical treatment of presumed Candida endocarditis in critically ill infants. Journal of Pediatrics, 119, 472-477. doi:10.1016/S0022-3476(05)82067-0

[12] Pappas, P.G. (2009) Clinical practice guidelines for the management of candidiasis: 2009 update by the infectious diseases society of America. Clinical Infectious Diseases, 48, 503-535. doi:10.1086/596757 